\title{
He Tamariki Kokoti Tau-Tackling Preterm: a data-linkage methodology to explore the clinical care pathway in preterm deliveries
}

Sara Filoche ${ }^{1 *}$ (D) Fiona Cram² ${ }^{2}$ Angela Beard ${ }^{3}$, Dalice Sim ${ }^{4}$, Stacie Geller ${ }^{5}$, Liza Edmonds ${ }^{6}$, Bridget Robson ${ }^{7}$ and Beverley Lawton ${ }^{8}$

\begin{abstract}
Background: Significant health inequities exist around maternal and infant health for Māori, the indigenous people of Aotearoa New Zealand - and in particular around a premature (preterm) delivery. Māori babies are more likely to be born preterm (8.1\%, compared to an overall rate of $7.4 \%)$ and they are more likely to have a preterm death. An essential part of redressing these disparities is to examine the clinical care pathway and outcomes associated with preterm deliveries. This paper describes a protocol utilising national and local health collections to enable such a study.

Design: This is a retrospective cohort study comprising 5 years data pertaining to preterm deliveries from 2010 to 2014. These data are generated from linked national administrative and local health information collections to explore a range of neonatal outcomes and infant mortality in relation to the antenatal care pathway and known risk factors for preterm delivery. This study is being conducted within a Kaupapa Māori paradigm that dismisses victim blaming and seeks to intervene at structural levels to improve the health and wellbeing of Māori whānau (family).

Significance of the study: Our data-linkage methodology optimises the utility of New Zealand health collections to address a significant health issue. Our findings will fill the information gaps around the burden of preterm delivery by quantifying the incidence of preterm delivery and adverse neonatal and infant outcomes in Aotearoa New Zealand. It will explore access to evidenced based care including use of steroids before birth, and appropriate place of delivery. The results from this study will inform maternity care services to improve management of preterm deliveries - both locally and internationally. This in turn will improve the preterm sequela by reducing the long-term health burden and health inequities.
\end{abstract}

Keywords: Indigenous health, Preterm delivery, Data linking, Equity, Clinical care pathway, Kaupapa Māori, Maternity care services, Health disparities

\section{Background}

Premature deliveries (preterm) represent a global health burden [1] for which there are significant disparities by ethnicity [2, 3]. In Aotearoa New Zealand such health inequities exist between Māori, the indigenous people of Aotearoa New Zealand, and Pākehā (New Zealand European) [4-7].

This paper describes the protocol for a retrospective cohort study that links local hospital, community laboratories and national health information datasets to explore the preterm delivery clinical care pathway, delivery and neonatal outcomes and infant mortality $(\leq 1$ yr. of age) of Māori and Pākehā (New Zealand European) women and their babies. Building on an approach we have previously described, $[8,9]$ we have further developed this methodology to enable an in-depth exploration of key elements of antenatal care such as the administration of corticosteroids in relation to neonatal and infant outcomes.

\footnotetext{
* Correspondence: Sara.filoche@otago.ac.nz

${ }^{1}$ Department of Obstetrics and Gynaecology and Department of Pathology and Molecular Medicine, University of Otago, Wellington, New Zealand Full list of author information is available at the end of the article
}

\section{Preterm deliveries in Aotearoa New Zealand}

A premature delivery (preterm) can potentially result in adverse outcomes such as death, brain haemorrhage, 
infection, chronic lung disease and long-term growth impairment and harm $^{40}$ including cerebral palsy, cognitive, visual and learning impairments [10-14]. Māori whānau (families) suffer a greater burden of this preterm harm and death than Pākehā. We have known of these disparities since Mantel's early work in the 1990s, with his findings repeatedly confirmed by others including Robson [7], Craig [15] and the Perinatal and Maternal Mortality Review Committee (PMMRC) [4]. Māori babies are most likely to be born preterm $(8.1 \%$, compared to an overall rate of $7.4 \%$ ) and they are more likely to have a preterm death [4]. There are approximately 1200 Mãori preterm births annually. Preterm is the second commonest cause of peri-natal death in Aotearoa accounting for $20 \%$ of deaths [4]. Māori have over twice the rate of preterm death compared to Pākehā (359 Māori babies died from 2007 to 2013 compared to 319 Pākehā) [4]. An essential part of redressing these disparities is to examine the clinical care pathway associated with preterm deliveries. This paper describes a protocol utilising local and national administrative health collections to enable such a study.

\section{Design}

This is a retrospective cohort study comprising 5 years data pertaining to preterm deliveries from 2010 to 2014 . These data are generated from linked national administrative and local health information collections to explore a range of neonatal outcomes and infant mortality in relation to the antenatal care pathway and known risk factors for preterm delivery such as the lack of antenatal corticosteroids prior to delivery or delivery at the appropriate care centre $[16,17]$.

This study is being conducted within a Kaupapa Māori paradigm that dismisses victim blaming and seeks to intervene at structural levels to improve the health and wellbeing of Māori whānau. It seeks to identify where resources may be best allocated to improve Māori health and wellbeing. Kaupapa Mãori Research is about ensuring good health outcomes for Māori and frames our approach [18-20].

\section{Outcome measures}

Primary outcomes

1. To compare for Māori and Pākehā the proportion for infants born preterm having at least one of the following outcomes:

- Death, up to 1 year post birth

- Brain injury (defined as periventricular and/or cerebellum haemorrhage and/or Periventricular Leucomalacia (PVL))
- Chronic lung disease of infancy (Bronchopulmonary BPD subtype)

- Oxygen and/or tube feeding on discharge

- Length of stay (LOS) (until first discharge home) at hospital $>4$ months

2. To compare for Māori and Pākehā the national rate of infants born preterm with at least one of the following outcomes: death, small for gestational age and chronic lung disease of infancy.

\section{Secondary outcomes}

To compare for Māori and Pākehā women the proportion of:

1. Women delivering preterm who receive full-course antenatal corticosteroid administration

2. The percentage of infants born preterm

3. Preterm infants not born at an appropriate level hospital (such as a tertiary hospital).

4. Preterm infants with retinopathy of prematurity and or deafness

5. Women who have a preterm delivery who receive appropriate antenatal screening

\section{Study population}

The study population includes pregnant women and their preterm babies born between 2010 and 2014 in Aotearoa New Zealand. For Primary Outcome 1, the study population includes pregnant women and their preterm infants born in 5 different district health board (DHBs) regions.

\section{Inclusion criteria}

All infants born to women between $24^{0}$ and $36^{6}$ completed week's gestation between January 1st 2010 and $31^{\text {st }}$ December 2014.

\section{Exclusion criteria}

Women not domiciled in the respective DHBs at the time of delivery (Primary Outcome 1 only). Infants less than 24 and greater than 36 weeks gestation will be excluded.

\section{Data sources \\ National health and clinical information collections in Aotearoa New Zealand}

The data for our study is sourced from routine health information collections at both national and local levels (Table 1). In Aotearoa New Zealand, the Ministry of Health is responsible for the oversight and funding of New Zealand's 20 DHBs. Select clinical information is reported by DHBs to the Ministry of Health, and is collated into national datasets with operational responsibility by the Client Insights and Analytics group. Among the wide range of health related registries and datasets 
Table 1 Local and national data sources and examples of variables collected

\begin{tabular}{|c|c|c|}
\hline Dataset & Type of information & Examples of variables extracted \\
\hline National health index & Sociodemographic & $\mathrm{NHI}$ number, area deprivation, ethnicity \\
\hline Mortality collection & Mortality & $\begin{array}{l}\text { Ethnicity, date of death, gestational age at termination of pregnancy, birth-weight, } \\
\text { diagnostic codes on cause of death, sudden and unexpected death indicator }\end{array}$ \\
\hline $\begin{array}{l}\text { National maternity } \\
\text { collection }\end{array}$ & $\begin{array}{l}\text { Pregnancy and } \\
\text { delivery information }\end{array}$ & $\begin{array}{l}\text { Maternal ethnicity, maternal height and weight at time of booking with a maternity care } \\
\text { provider, maternal smoking status at time of booking with maternity care provider, } \\
\text { plurality, parity, mode of delivery, Apgar at } 5 \text { min, birth weight, gestational age at delivery }\end{array}$ \\
\hline $\begin{array}{l}\text { National minimum } \\
\text { dataset }\end{array}$ & Hospital events & $\begin{array}{l}\text { Ethnicity, maternal and/or infant hospital admissions (public) and discharge dates, } \\
\text { length of stay, diagnostic (ICD-10) codes }\end{array}$ \\
\hline $\begin{array}{l}\text { Australia New Zealand } \\
\text { Neonatal network }\end{array}$ & Neonatal intensive care & $\begin{array}{l}\text { Ethnicity, corticosteroid administration before delivery, length of stay, neonatal surgery, } \\
\text { corrected gestational age on discharge, oxygen on discharge, enteral tube feeding on } \\
\text { discharge (e.g. nasogastric tube) }\end{array}$ \\
\hline $\begin{array}{l}\text { Local hospital } \\
\text { (DHB specific) }\end{array}$ & Pregnancy and delivery events & $\begin{array}{l}\text { Maternal and paternal ethnicity, obstetric history, plurality, parity, date of admission, } \\
\text { date of delivery }\end{array}$ \\
\hline Community laboratory & Screening and diagnostics & $\begin{array}{l}\text { Antenatal booking screening (e.g. blood count), gestational diabetes screening, } \\
\text { sexually transmitted infection (note: only if screened and not results) }\end{array}$ \\
\hline
\end{tabular}

held in New Zealand are: National Minimum Data Set (NMDS, covering hospital discharges), Mortality Collection, and National Maternity Collection (MAT) [21].

In addition, there is an Australia New Zealand Neonatal Network (ANZNN) collection which is a collaboration of every neonatal intensive care unit in the two countries, established in 1994 [22]. The inclusion criteria for registration in this dataset are babies admitted to a neonatal unit who meet one or more of the following:

- born at less than 32 weeks gestation, or

- weighed less than $1500 \mathrm{~g}$ at birth, or

- received assisted ventilation (mechanical ventilation) including intermittent positive pressure ventilation (IPPV) or continuous positive airways pressure (CPAP) or high flow nasal cannulae for four or more consecutive hours, or died while receiving mechanical ventilation prior to 4 hours of age, or

- received major surgery (surgery that involved opening a body cavity), or

- received therapeutic hypothermia

\section{Local health and clinical information collections in Aotearoa New Zealand}

Clinical information that is not reported to the Ministry of Health (so does not appear in these national collections) is held locally within independent information systems maintained within each DHB: for example the Perinatal Information Management System (PIMS), which collects information on perinatal events (Table 1).

In Aotearoa community laboratories offer a range of services; taken as indicator/proxy of receiving appropriate antenatal care (as these tests are usually carried out when the woman registers with her maternity care provider and during pregnancy) and relevant to this study include antenatal blood tests such as blood count and gestational diabetes screening.

\section{Data collection and matching}

The variables retrieved from each collection (as described in the associated data dictionaries) were discussed in consultation with the research team and were informed by the Client Insights and Analytics group who were able to provide information pertaining to the quality of data (e.g. missing data). Risk factor variables (confounders for analysis) for preterm delivery were also discussed in-line with available data and where possible were collected (e.g. maternal smoking status) or constructed (e.g. inter-timing pregnancy and previous preterm delivery).

For Primary Outcome 2, two datasets, pregnancy information and fetal-neonatal outcome information, are joined using the unique pregnancy identifier as outlined in Fig. 1 (and cross referenced with hospitalisation discharge information as a quality checking step). The pregnancy identifier is unique for each pregnancy event, and enables clear delineation of each pregnancy and fetal-neonatal outcome information. For example, if a pregnancy is multiparous e.g. twin, in the pregnancy dataset the identifier would appear once and twice in the fetal-neonatal outcome dataset. Using a combination of linking approaches with infant and maternal NHI numbers, the pregnancy and fetal-neonatal outcome dataset are linked to the hospitalisation discharge information (which contains ICD-10 codes) and mortality collection.

Based on domicile status from the combined pregnancy-fetal-neonatal dataset we have identified relevant pregnancies for Primary Outcome 1 (Fig. 1). Using maternal NHIs the community laboratory and hospital data are arranged so that each line in the dataset 


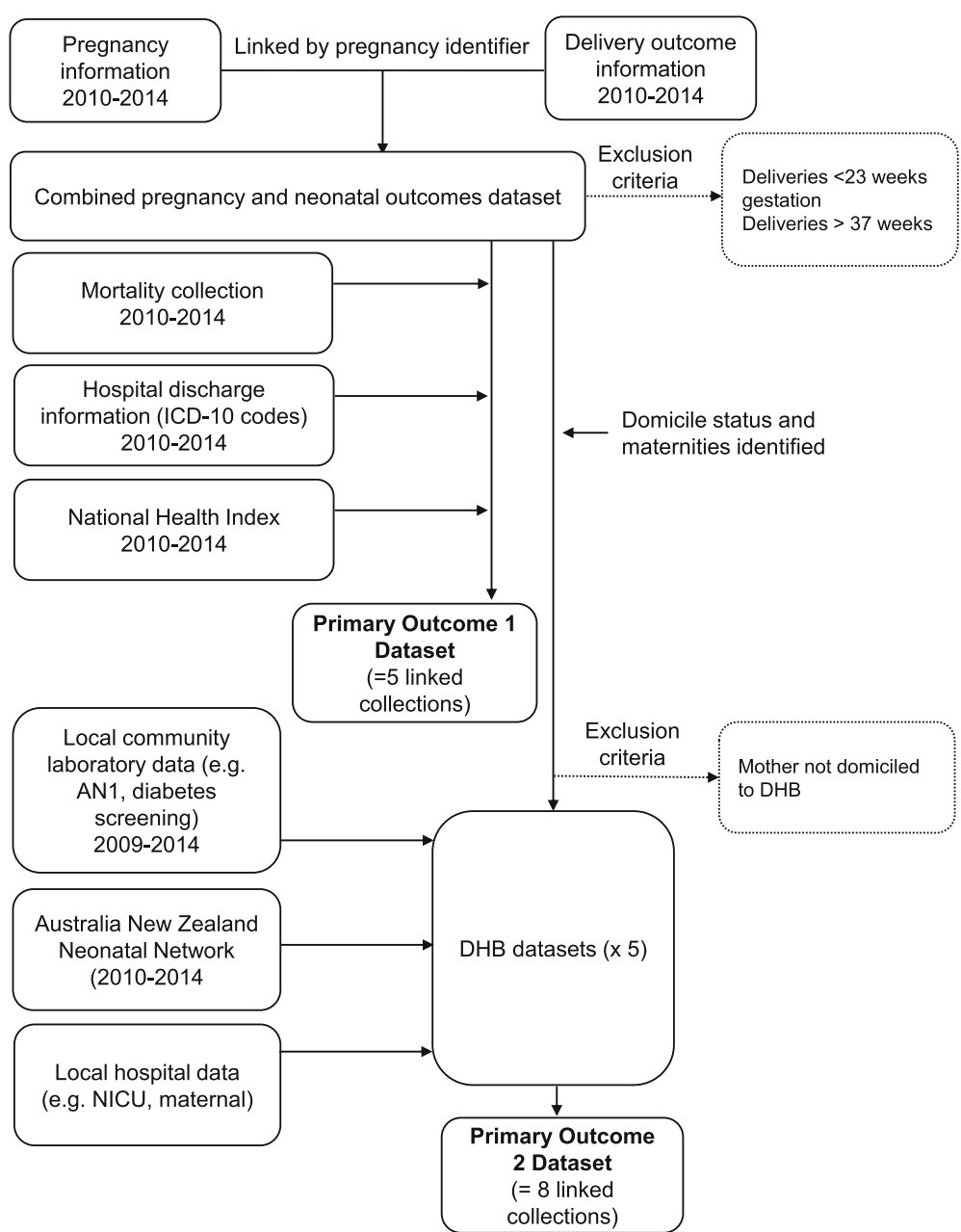

Fig. 1 Overview of study protocol outlining the main data collation steps and exclusion criteria for each Primary OutcomeAN1 (Antenatal booking).NICU (Neonatal Intensive Care Unit)

represents pregnancy information with fetal-neonatal outcome and also antenatal care-pathway elements of interest for that pregnancy. For example, whether the mother received gestational diabetes screening (which comprises a constructed variable flagged as yes or no; and is calculated based on whether screening occurred at the appropriate time according to guidelines at the time and at any time during pregnancy). In order to capture the antenatal screening data for deliveries in 2010, data from community laboratories were extracted from 2009.

Each collection is systematically explored for missing variables, and "cleaned" to remove any duplicate entries before any dataset linking or any variables are constructed e.g. number of repeat pregnancies and intertiming pregnancy.

No personally identifiable data (i.e. names, addresses) have been or will be extracted from any of the data sources.

\section{Statistical analysis}

In Aotearoa New Zealand there are approximately 60,000 live births per annum which for 5 years data would equate to 300, 000 delivery events. Based on a national average of preterm deliveries of 7.5\% [4] our estimated population will comprise approximately 21, 000 preterm delivery events.

Sample size estimates are presented for Primary Outcome 1. To estimate the number of Māori and Pākehā babies born preterm per year between 2010 and 2014 we used annual maternity data on births for each of the 5 DHBs, and based the proportion of births that were preterm for Māori and Pākehā on a tertiary level DHB (approximately 11.7\%, $65 / 559$ cases of preterm birth per annum for Māori and 8.1\%, 270/3344 for Pākehā mothers). Using these assumptions, there are approximately 654 Maori preterm births and 1236 Pākehā preterm births per year across the 5 DHBs. Working 
from a baseline assumption that the preterm infants of Pākehā women will have an $8 \%$ rate of poor outcomes (ANZNN overall rate for chronic lung disease in infants at 36 weeks) [23, 24] with 1 year's worth of data, this study will have $80 \%$ power (using an alpha of 0.05 ) to detect a significantly enhanced risk if poor outcomes are $11.7 \%$ or higher among Māori infants. If 2 years' worth of data is used (1308 Māori preterm births and 2470 Pākehā preterm births), the study will have $80 \%$ power (using an alpha of 0.05 ) to detect a significantly higher risk if poor outcomes are $10.5 \%$ or higher in Māori infants.

For Primary Outcome 1 multivariable Poisson regression models will compare the rates of poor outcomes between preterm infants of Māori and Pākehā mothers, after adjusting for potential personal and care-related confounders [25-27] e.g. maternal age, rapid repeat pregnancies, antenatal screening, parity, socioeconomic status and smoking, and to account for the degree to which ethnic disparities in infant health outcomes are mediated by these factors. Descriptions of rates, rate ratios and respective $95 \%$ confidence intervals will be reported.

\section{Discussion}

This is a multi-disciplinary collaborative study that aims to bring about positive changes in antenatal care for women at risk of having preterm delivery, and consequently for their infants.

Our data-linkage methodology optimises the utility of New Zealand health collections to address a significant health issue $[1,28,29]$. Our findings will fill the information gaps around the burden of preterm delivery by quantifying incidence of preterm delivery and adverse neonatal and infant outcomes in Aotearoa New Zealand [30]. It will explore access to evidence based care including use of steroids before birth, and appropriate place of delivery. The results from this study will inform maternity care services, both nationally and internationally. This study aligns with the New Zealand government goals to deliver quality health services, improve system performance, and reduce disparities in health. A complimentary qualitative study exploring the experiences of Māori whānau whose newborn has been admitted to neonatal intensive care because of a preterm delivery is also being carried out alongside this investigation (He Tamariki Kokoti Tau-Addressing Preterm).

The described study utilises both local and national level data collections and through a process of linking by unique identifiers is able to quantitatively explore and analyse the preterm care pathway and its outcomes. We are informed both by clinical expertise in the area of obstetrics and neonatalogy - both from the research team and local investigators. We are also informed by our kaumātua (Māori elders). The analysis for this study is still underway. It is expected that this in-depth investigation from a mother to infant perspective will provide a greater understanding of the clinical care pathway in preterm deliveries, which will inform local national health care policy and procedures around such maternities. As such maternity care providers will be able to identify high risk patients, intervene earlier and provide more appropriate management. This will in turn reduce the health burden of being "born too soon" [29] and also redress the health inequity associated with preterm deliveries, both within Aotearoa New Zealand and globally.

\section{Abbreviations}

ANZNN: Australia New Zealand Neonatal Network; CPAP: Continuous positive airways pressure; DHB: District health board; IPPV: Intermittent positive pressure ventilation; LOS: Length of stay; MAT: National maternity collection; NICU: Neonatal intensive care unit; NMDS: National minimum data set; PIMS: Perinatal information management system; PMMRC: Perinatal and maternal mortality review committee; PVL: Periventricular Leucomalacia

\section{Acknowledgements}

This research has been funded by the Health Research Council of New Zealand (16-444). The authors thank the staff at the Client Insights and Analytics Group at the Ministry of Health and the local research and clinicians for their input and support for the study.

\section{Funding}

This study is funded by the Health Research Council of New Zealand (HRC 16-444)

\section{Availability of data and materials}

The datasets generated and/or to be analysed during the current study are not publicly available as this is not part of our ethical approval.

\section{Authors' contributions}

SF drafted the manuscript and is responsible for leading development of data linking methodology, the acquisition of data and data cleaning, database management and interpretation of the data. FC contributed to study development, methodology and manuscript preparation. $A B$ contributed to study development, methodology and manuscript preparation. DS provided statistical input, study design manuscript preparation. SG contributed to study development, methodology and manuscript preparation. LE contributed to study development, methodology and manuscript preparation. BR contributed to study development, methodology, data interpretation and manuscript preparation. BL conceived of the study including rationale, outcomes, contributed to methodology, manuscript and has overall responsibility for the study. All authors read and approved the final manuscript.

\section{Ethics approval and consent to participate}

Ethics approval is needed for this study as we are using un-encrypted NHI numbers (to enable accurate individual level matching from the mother to fetal/neonatal outcomes). Ethics approval to utilise data from the national collections is obtained through the Health and Disability Ethics Committee (HDEC). Our study has been granted national ethical approval by the New Zealand Northern B Ethics Committee (Ref 16/NTB/186).

Local ethics approval is needed for the in-depth study in the 5 DHBs to obtain and use local hospital data. Each DHB has its own process which also comprises an application to local Māori research committees to help ensure that the research is tika (ethical) and is of benefit to Māori. Face-to-face consultation with key stakeholders is carried out at each DHB prior to application. The application to grant permission to obtain and use local hospital data is discussed at respective committees such as the Women's Health Governance Group, and it is usual for a clinician to be named as a local investigator, this ensures that the study meets local requirements and to provide appropriate interpretation of the results (i.e. are informed by local knowledge). Results will not be published without prior knowledge of the relevant stakeholders. 


\section{Competing interests}

The authors declare they have no competing interests.

\section{Publisher's Note}

Springer Nature remains neutral with regard to jurisdictional claims in published maps and institutional affiliations.

\section{Author details}

'Department of Obstetrics and Gynaecology and Department of Pathology and Molecular Medicine, University of Otago, Wellington, New Zealand. ${ }^{2}$ Katoa Ltd, Auckland, New Zealand. ${ }^{3}$ Christchurch Obstetric Associates, Christchurch, New Zealand. ${ }^{4}$ Dean's Department, University of Otago, Wellington, New Zealand. ${ }^{5}$ Department of Obstetrics and Gynecology, University of Illinois, Chicago, USA. 'Women's and Children's Health Paediatrics \& Child Health, Dunedin School of Medicine, Health Sciences and Southern District Health Board, Dunedin, New Zealand. ${ }^{7} T e$ Rōpū Rangahau Hauora a Eru Pōmare, University of Otago, Wellington, New Zealand. ${ }^{8}$ Centre for Women's Health Research-Te Tātai Hauora O Hine, Faculty of Health, Victoria University of Wellington, Wellington, New Zealand.

Received: 28 November 2017 Accepted: 2 May 2018

Published online: 21 May 2018

\section{References}

1. Harrison MS, Goldenberg RL. Global burden of prematurity. Semin Fetal Neonatal Med. 2016;21(2):74-9.

2. Burris HH, Collins JW, Wright RO. Racial/ethnic disparities in preterm birth clues from environmental exposures. Curr Opin Pediatr. 2011;23(2):227-32.

3. Smid MC, Lee JH, Grant JH, Miles G, Stoddard GJ, Chapman DA, Manuck TA. Maternal race and intergenerational preterm birth recurrence. Am J Obstet Gynecol. 2017;217(4):480.e481-9.

4. Perinatal Maternal Mortality Review Committee. Seventh annual report of the perinatal and maternal mortality review committee: reporting mortality 2011. Wellington: Health Quality and Safety Commission; 2013.

5. Ajwani S, Blakely T, Robson B, Bonne M, Tobias M. Decades of disparity: ethnic mortality trends in New Zealand 1980-1999. Ministry of Health: Wellington, New Zealand; 2003.

6. Hill S, Sarfati D, Blakely T, Robson B, Purdie G, Chen J, Dennett E, Cormack $D$, Cunningham R, Dew K, et al. Survival disparities in indigenous and nonindigenous new Zealanders with colon cancer: the role of patient comorbidity, treatment and health service factors. J Epidemiol Community Health. 2010;64(2):117-23.

7. Robson B, Harris R. Hauora: Māori standards of health IV. A study of the years 2000-2005. Te Rōpū Rangahau Hauora a Eru Pōmare: Wellington; 2007.

8. Filoche S, Garrett S, Stanley J, Rose SB, Robson B, Elley CR, Lawton B. Wahine hauora: linking local hospital and national health information datasets to explore maternal risk factors and obstetric outcomes of New Zealand Māori and non-Māori women in relation to infant respiratory admissions and timely immunisations. BMC Pregnancy Childbirth. 2013;13:145.

9. Lawton B, Stanley J, Filoche SK, Garrett S, Rose SB, Robson B, Raina EC. Exploring the maternal infant continuum - ethnic disparities in infant hospital admissions for respiratory disease: a Kaupapa Māori retrospective cohort study. Aust N Z J Public Health, In press. 2015;

10. Bird TM, Bronstein JM, Hall RW, Lowery CL, Nugent R, Mays GP. Late preterm infants: birth outcomes and health care utilization in the first year. Pediatrics. 2010;126(2):e311-9.

11. Saigal S, Doyle LW. An overview of mortality and sequelae of preterm birth from infancy to adulthood. Lancet. 2008:371(9608):261-9.

12. Swamy GK, Ostbye T, Skjaerven R. Association of preterm birth with longterm survival, reproduction, and next-generation preterm birth. JAMA. 2008; 299(12):1429-36.

13. Carr H, Cnattingius S, Granath F, Ludvigsson JF, Edstedt Bonamy AK Preterm birth and risk of heart failure up to early adulthood. J Am Coll Cardiol. 2017;69(21):2634-42.

14. Ludwig CA, Chen TA, Hernandez-Boussard T, Moshfeghi AA, Moshfeghi DM. The epidemiology of retinopathy of prematurity in the United States. Ophthalmic Surg Lasers Imaging Retin. 2017:48(7):553-62.

15. Craig ED, Mitchell EA, Stewart AW, Mantell CD, Ekeroma AJ. Ethnicity and birth outcome: New Zealand trends 1980-2001: part 4. Pregnancy outcomes for European/other women. Aust N Z J Obstet Gynaecol. 2004;44(6):545-8.
16. American College of Obstetricians and Gynecologists. Antenatal corticosteroids for accelerating fetal lung maturation for women at risk of preterm birth. Obstet Gynecol. 2007;109(1):189-90.

17. Chien L, Whyte R, Aziz K, Thiessen P, Matthew D, Lee S. Canadian neonatal network: improved outcome of preterm infants when delivered in tertiary care centers. Obstet Gynecol Clin N Am. 2001;98(2):247-52.

18. Keefe V, Ormsby C, Robson B, Reid P, Cram F, Purdie G. Kaupapa Māori meets retrospective cohort. He Pukenga Körero. 1999;5:12-7.

19. Lawton B, Cram F, Makowharemahihi C, Ngata T, Robson B, Brown S, Campbell W. Developing a Kaupapa Māori research project to help reduce disparities experienced by young Māori women and their babies. AlterNative. 2013;9(3):246-61

20. Cram F. Kaupapa Māori health research. In: Handbook of research methods in health and social sciences. Switzerland: Springer International Publishing; 2017.

21. National collections and surveys [http://www.health.govt.nz/nz-healthstatistics/national-collections-and-surveys].

22. Improving clinical practice through collaboration [https:/anznn.net/]

23. Chow SSW. Report of the Australian and New Zealand neonatal network 2011. Sydney: ANZNN; 2013.

24. Australian and New Zealand Neonatal Network. Report of the Australian and New Zealand neonatal network 2006. Sydney: ANZNN; 2009.

25. Mortier I, Blanc J, Tosello B, Gire C, Bretelle F, Carcopino X. Is gestational diabetes an independent risk factor of neonatal severe respiratory distress syndrome after 34 weeks of gestation? A prospective study. Arch Gynecol Obstet. 2017;296(6):1071-7. https://doi.org/10.1007/s00404-017-4505-7.

26. Phillips JK, Skelly JM, King SE, Bernstein IM, Higgins ST. Associations of maternal obesity and smoking status with perinatal outcomes. J Matern Fetal Neonatal Med. 2017;31(12):1620-6. https://doi.org/10.1080/14767058.

27. Yu L, Zeng XL, Cheng ML, Yang GZ, Wang B, Xiao ZW, Luo X, Zhang BF, Xiao DW, Zhang S, et al. Quantitative assessment of the effect of pregestational diabetes and risk of adverse maternal, perinatal and neonatal outcomes. Oncotarget. 2017;8(37):61048-56

28. Chang HH, Larson J, Blencowe H, Spong CY, Howson CP, Cairns-Smith S, Lackritz EM, Lee SK, Mason E, Serazin AC, et al. Preventing preterm births: analysis of trends and potential reductions with interventions in 39 countries with very high human development index. Lancet. 2013; 381(9862):223-34

29. March of Dimes, PMNCH, Save the Children, WHO. In: Howson MK CP, Lawn JE, editors. Born too soon: the global action report on preterm birth. Geneva: World Health Organization; 2012.

30. Committee NZPHA. Improving child oral health and reducing child oral health inequalities. National Advisory Committee on Health and Disability: Wellington; 2003.

\section{Ready to submit your research? Choose BMC and benefit from:}

- fast, convenient online submission

- thorough peer review by experienced researchers in your field

- rapid publication on acceptance

- support for research data, including large and complex data types

- gold Open Access which fosters wider collaboration and increased citations

- maximum visibility for your research: over $100 \mathrm{M}$ website views per year

At BMC, research is always in progress.

Learn more biomedcentral.com/submissions 